\title{
Respuestas cardiovasculares de mujeres con obesidad mórbida sometidas a un test ergoespirométrico con ergómetro de brazo
}

\author{
Felipe Monnerat Marino Rosa ${ }^{\mathrm{a}, \mathrm{b}, *}$, Renato Vidal Linhares ${ }^{\mathrm{a}, \mathrm{d}}$, \\ Luiz Fernando Parada Martinez ${ }^{a}$, José Carlos do Valle Quaresma ${ }^{b}$, \\ Roberto Fares Simão ${ }^{c}$, João Regis Ivar Carneiro ${ }^{b}$, Valéria Bender Braulio ${ }^{b}$ \\ y José Fernandes Filho ${ }^{a, c}$
}

a Laboratorio de Biociencias Humanas, Universidad Federal de Río de Janeiro, Río de Janeiro, Brasil
b Hospital Clementino Fraga Filho, Universidad Federal de Río de Janeiro, Río de Janeiro, Brasil
'Escuela de Educación Física y Deportes, Universidad Federal de Río de Janeiro, Río de Janeiro, Brasil
d Grupo de Estudos em Educação Física Escolar do Colégio Pedro Il, Río de Janeiro, Brasil

Recibido el 4 de mayo de 2016; aceptado el 9 de mayo de 2017

Disponible en Internet el 31 de agosto de 2017

\section{PALABRAS CLAVE Obesidad mórbida; Frecuencia cardíaca; Presión arterial; Doble producto; Prueba de esfuerzo}

\begin{abstract}
Resumen
Introducción: la obesidad mórbida es una enfermedad de etiología multifactorial, de gran prevalencia en el mundo, que guarda relación directa con otras comorbilidades.

Objetivo: analizar las respuestas cardiovasculares de pacientes mujeres con obesidad mórbida, a través de un test de esfuerzo máximo para la estratificación del riesgo y la prescripción de ejercicio.

Método: se incluyeron trece pacientes con obesidad grado III, de sexo femenino, con edad entre 20 a 40 años, que fueron sometidas a un test ergoespirométrico en el cicloergómetro de miembros superiores para la verificación y el análisis de la frecuencia cardiaca, la presión arterial y el doble producto.

Resultados: las pacientes con media de edad de 34,7 $\pm 5,1$ años tenían índice de masa corporal de $46,5 \pm 3,8 \mathrm{~kg} / \mathrm{m}^{2}$. Alcanzaron una frecuencia cardíaca máxima de $168,2 \pm 14,6$ lpm, sin respuesta hipertensiva al esfuerzo, y presión arterial de $171,1 \pm 22,2 \mathrm{~mm} \mathrm{Hg}$ (sistólica) $\times 87,5 \pm 4,2 \mathrm{~mm} \mathrm{Hg}$ (diastólica). El doble producto máximo encontrado fue de $28.662,2$ $\pm 3.644,1 \mathrm{~mm} \mathrm{Hg.lpm}$.

Conclusión: de acuerdo con los resultados de este estudio se observó una reducción de la frecuencia cardiaca, la presión arterial y el doble producto cardiaco, compatible con el esfuerzo esperado para el test.

(C) 2017 Sociedad Colombiana de Cardiología y Cirugía Cardiovascular. Publicado por Elsevier España, S.L.U. Este es un artículo Open Access bajo la licencia CC BY-NC-ND (http:// creativecommons.org/licenses/by-nc-nd/4.0/).
\end{abstract}

\footnotetext{
* Autor para correspondencia.

Correo electrónico: felipemonnerat@gmail.com (F.M. Marino Rosa).
} 


\section{KEYWORDS}

Morbid obesity;

Heart rate;

Blood pressure;

Double product;

Stress test

\section{Cardiovascular responses of morbid obese women subjected to an ergospirometry test with an arm ergometer}

\begin{abstract}
Introduction: Morbid obesity is a condition of multifactorial origin with a high prevalence worldwide, and has a direct relationship with other illnesses.

Objective: To analyse the cardiovascular responses of morbid obese women using a maximum stress test for stratifying risk and the prescribing of exercise.

Method: The study included 13 patients with Grade III obesity. All were women between 20 and 40 years of age. Their arms were subjected to an ergospirometry test on the cycle ergometry in order to check and analyse heart rate, blood pressure, and the double product.

Results: The patients had a mean age of $34.7 \pm 5.1$ years, and a mean body mass index of $46.5 \pm 3.8 \mathrm{~kg} / \mathrm{m}^{2}$. A maximum heart rate of $168.2 \pm 14.6 \mathrm{bpm}$ was achieved, with no hypertensive response to the stress, and blood pressure of $171.1 \pm 22.2 \mathrm{~mm} \mathrm{Hg}$ (systolic) $\times 87.5 \pm 4.2 \mathrm{~mm}$ $\mathrm{Hg}$ (diastolic). The maximum double product found was $28,662.2 \pm 3,644.1 \mathrm{~mm} \mathrm{Hg}$.bpm.

Conclusion: In the results of this study is observed reduction of heart rate, blood pressure, and the double cardiac product, compatible with the expected effort for the test.

(c) 2017 Sociedad Colombiana de Cardiología y Cirugía Cardiovascular. Published by Elsevier España, S.L.U. This is an open access article under the CC BY-NC-ND license (http:// creativecommons.org/licenses/by-nc-nd/4.0/).
\end{abstract}

\section{Introducción}

La obesidad, reconocida en el mundo como enfermedad crónica $^{1}$, se caracteriza por la acumulación excesiva de tejido adiposo que genera complicaciones para la salud ${ }^{1,2}$ y es de etiología multifactorial ${ }^{1-5}$. La Organización Mundial de la Salud (OMS) adopta el índice de masa corporal (IMC) para clasificar la obesidad; valores encima de $40 \mathrm{~kg} / \mathrm{m}^{2}$ corresponden a la forma más grave, obesidad grado III u obesidad mórbida ${ }^{1,3,6}$. Esta última, además de ser una enfermedad y generar complicaciones, generalmente acarrea comorbilidades, entre ellas hipertensión arterial, dislipidemias, enfermedad pulmonar obstructiva crónica (EPOC), cardiopatías, cáncer ${ }^{1,3,5,7,8}$, estrés y ansiedad ${ }^{2,4,9}$, sumado a la dificultad de locomoción debido al exceso de peso ${ }^{7,10}$. En este sentido, el cicloergómetro de miembros superiores presenta buena aceptación y está indicado por diversos autores en parapléjicos ${ }^{11}$, cardiópatas y obesos ${ }^{11-13}$.

En la actualidad, en la práctica clínica se utilizan los valores de frecuencia cardíaca y presión arterial para seguridad cardiovascular y prescripción del ejercicio; entretanto, para individuos hipertensos, adultos mayores, obesos y diabéticos es necesaria la evaluación del doble producto ${ }^{14,15,27}$, un método no invasivo que evalúa la sobrecarga cardiaca en reposo o durante el esfuerzo y tiene una fuerte correlación con el consumo de oxígeno del miocardio $\left(\mathrm{MVO}_{2}\right)$, siendo estimado por el producto entre la presión arterial sistólica y la frecuencia cardiaca ${ }^{14,16}$. Se consideran máximos los valores obtenidos en la última fase del esfuerzo o durante el esfuerzo máximo, es decir, en la mayor frecuencia cardiaca y presión arterial sistólica alcanzadas ${ }^{17}$. El doble producto puede ser considerado un indicador fidedigno del trabajo del corazón durante esfuerzos físicos continuos y es de gran importancia en la evaluación del dolor torácico y de la isquemia miocárdica ${ }^{17}$; adicionalmente, la correlación entre el doble producto y $\mathrm{MVO}_{2}$ es de casi $90 \%$ y por esa razón el doble producto se convierte en una variable relevante en el acompañamiento y la prescripción de ejercicios en personas que necesitan cuidados.

El objetivo del estudio fue analizar las respuestas cardiovasculares de pacientes mujeres con obesidad mórbida a través de un test de esfuerzo máximo para la estratificación del riesgo y prescripción del ejercicio.

\section{Métodos}

\section{Aspectos éticos}

El estudio fue aprobado por el Comité de Ética de la Universidad Federal de Río de Janeiro-RJ, protocolo No. 12520413.6.0000.5257. Todas las participantes firmaron el Acuerdo de Consentimiento Libre y Explicativo (ACLE). El trabajo fue desarrollado en el Hospital Universitario Clementino Fraga Filho, en los laboratorios de Nutrición y Biociencias de la motricidad humana.

\section{Muestra}

Se optó por una muestra por conveniencia, considerando como población de estudio, todas las mujeres con obesidad grado III, atendidas por el Programa de Cirugía Bariátrica en el Hospital de la Universidad Federal de Río de Janeiro. Como criterios de inclusión y exclusión se consideraron los siguientes: estar entre 20 a 40 años, tener IMC entre 40 y $60 \mathrm{~kg} / \mathrm{m}^{2}$, no padecer enfermedad pulmonar obstructiva, sin uso de insulina, medicación betabloqueadores y en fase preoperatoria de cirugía bariátrica. Se incluyeron en total 13 pacientes. 
Tabla 1 Presión arterial sistólica y diastólica de mujeres con obesidad de grado III en un test ergoespirométrico en cicloergómetro de miembros superiores

\begin{tabular}{lcccc}
\hline & Media & Desviación estándar & Mínimo & Máximo \\
\hline PAS reposo & 130,2 & 6,85 & 120 & 142 \\
PAS máxima & 171,1 & 22,15 & 150 & 210 \\
PAS recuperación 3' & 139,9 & 12,28 & 120 & 160 \\
PAS recuperación 5' & 128,8 & 8,51 & 114 & 76 \\
PAD reposo & 84 & 5,57 & 80 & 96 \\
PAD máxima & 87,5 & 4,18 & 76 & 94 \\
PAD recuperación 3' & 85,6 & 5,91 & 80 & 95 \\
PAD recuperación 5' & 85,1 & 4,94 & & 92 \\
\hline
\end{tabular}

PAS: presión arterial sistólica, PAD: presión arterial diastólica.

Tabla 2 Frecuencia cardíaca de mujeres con obesidad grado III durante un test ergoespirométrico en un cicloergómetro de miembros superiores

\begin{tabular}{lclrr}
\hline & Media & Desviación estándar & Mínimo & Máximo \\
\hline FC reposo & 83,7 & 10,2 & 65 & 97 \\
FC máxima & 168,2 & 14,51 & 145 & 193 \\
FC recuperación 1' & 155 & 1,81 & 102 & 159 \\
FC recuperación 3' & 128,8 & 8,51 & 96 & 144 \\
FC recuperación 5' & 84 & 5,57 & 93 & 136 \\
\hline
\end{tabular}

FC: frecuencia cardíaca.

\section{Ergómetro y espirómetro}

Se utilizó un cicloergómetro de brazo de la marca TECHNOGYM, modelo EXCITE PRÓ con freno electromagnético. El ergómetro posee un ajuste en el asiento, que aproxima o aleja al paciente de las manivelas, otro diseñado para los brazos de las manivelas, a fin de evitar la extensión completa del codo durante el movimiento, y un ajuste para palanca, que permite optar por un agarre pronado, neutro o semipronado; adicionalmente, existe un último ajuste para la altura de las manivelas, de modo que los hombros permanecen en la misma altura del eje de la manivela. Se usó el sistema analizador de gases y calorímetro (espirómetro) VMax $29 \mathrm{~N}$ Encore de CAREFUSION.

\section{Protocolo}

Se aplicó el protocolo de Rampa, mediante la fórmula de Shvartz y Rabold, para cicloergómetro de miembros inferiores $\mathrm{VO}_{2}=40,31-(0,41 \times$ edad $)$, corregida para miembros superiores (menos $35 \%$ del $\mathrm{VO}_{2 \text { máx }}$ esperado), y se consideró la diferencia entre ambos ergómetros ${ }^{18,19}$. El resultado se basó en la fórmula de la ACSM para el cálculo de la potencia máxima en el cicloergómetro de miembros superiores $\mathrm{VO}_{2 \text { máx }}=\mathrm{W}_{\text {máx }} \times 18,36+(\text { peso } \times 3,5)^{19}$, reduciendo $50 \%$ de la potencia máxima encontrada, de acuerdo con la propuesta de la tercera directriz de la Sociedad Brasileña de Cardiología para el test ergométrico ${ }^{20}$. El cálculo para la frecuencia máxima prevista se hizo a través de la fórmula propuesta por Sheffield et al.: $\mathrm{FC}_{\text {máx }}=198-(0,42 \times \text { edad })^{19}$. La frecuencia cardiaca se evaluó durante todo el test a través de un electrocardiograma marca TEB, mientras que la presión arterial sistólica y diastólica y el doble producto se valoraron durante reposo de cinco minutos en la posición sentado, después del momento máximo de esfuerzo y durante el periodo de recuperación con tres y cinco minutos.

La presión arterial sistólica y diastólica se midieron con ayuda de un estetoscopio de la marca GLICOMED modelo CARDIOLOGY y un esfigmomanómetro aneroide de la marca GLICOMED, en el brazo derecho del paciente, con una abrazadera de $33-44 \mathrm{~cm}$, específica para obesos mórbidos. El doble producto se estimó a través de la multiplicación de la frecuencia cardiaca por la presión arterial sistólica.

\section{Tratamiento estadístico}

En este estudio se aplicó estadística descriptiva para las variables colectadas, organizando las medidas de tendencia central (media), valores mínimos, máximos y variabilidad (desviación estándar) en tablas. Para mejor entendimiento se diseñaron gráficos en líneas, mediante el software Excel versión 2010.

\section{Resultados}

Los resultados se presentan en las tablas 1 y 2 y figura 1 .

\section{Discusión}

El test realizado en miembros superiores generalmente causa un aumento más expresivo de la presión arterial que un test realizado en miembros inferiores ${ }^{21}$, debido a la vasoconstricción de otros grupos ${ }^{21}$. La resistencia periférica es más elevada que en el test con miembros inferiores ${ }^{11,13,22}$ y hay dos factores que contribuyen a una mayor resistencia 


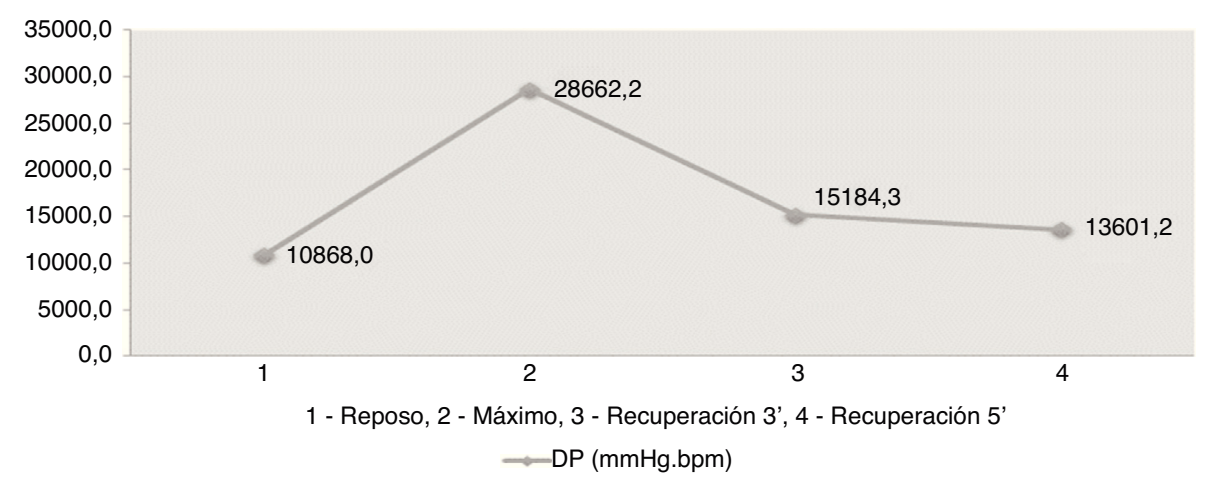

Figura 1 Doble producto cardíaco de pacientes obesas grado III sometidas a un test ergoespirométrico en cicloergómetro de miembros superiores. El eje Y representa el doble producto (PAS x FC) en (mm Hg.lpm) y eje X: 1 - Reposo, 2-Máximo, 3-Después de 3 minutos de recuperación y 4-después de 5 minutos de recuperación.

periférica durante el ejercicio con miembros superiores: la mayor secreción relativa de catecolaminas y la contracción isométrica necesaria para la estabilización del tronco ${ }^{11}$. En este trabajo se verificó que después del aumento de la presión arterial sistólica en el test, ésta volvió a valores basales luego de cinco minutos de reposo, mientras que la presión arterial diastólica media aumentó durante el ejercicio realizado con miembros superiores, hecho que sugiere un mayor componente isométrico.

Para confiabilidad de la medida intraevaluador se tomaron tres medidas de la presión arterial en reposo en diez pacientes con obesidad grado III a un intervalo de diez minutos entre medidas. La comparación de las medidas de presión arterial sistólica y diastólica se hizo a través de ANOVA ( $p=0,9893$ y $p=0,9946$, respectivamente); no se encontró diferencia estadísticamente significativa entre las mediciones realizadas por el evaluador en cuanto a las medidas referidas, para $p>0,05$.

En todas las pacientes hubo un aumento lineal de la frecuencia cardíaca debido al incremento del débito cardiaco y de la masa muscular involucrada ${ }^{23}$. Al iniciar el ejercicio, el sistema nervioso autónomo propicia la elevación del retorno de sangre al corazón, ocasionando mayor distensión del ventrículo derecho para recibir el mayor número de sangre, sumado a la taquicardia que ocurre posteriormente y al aumento del débito cardiaco ${ }^{24}$.

Todas las pacientes alcanzaron un $85 \%$ de la frecuencia cardiaca máxima, pero la mayoría no alcanzó la máxima prevista para el test debido a fatiga de los miembros superiores, especificidad del test y sedentarismo. Se observó reducción de la frecuencia cardiaca superior al $20 \%$ en el primer minuto de recuperación, comparada con la frecuencia de pico, dato importante, pues la reducción de la frecuencia cardiaca en el primer minuto de recuperación posibilita inferir la modulación vagal cardiaca: reducciones iguales o inferiores a 12 latidos por minuto tienen una asociación con mayor prevalencia de mortalidad ${ }^{25}$. Seres et al. compararon 31 obesos mórbidos con 30 sujetos eutróficos en un test de estera y verificaron que durante el esfuerzo los obesos presentaron mayor frecuencia cardiaca y mayor presión arterial sistólica que el grupo control. Sin embargo, después de la corrección del consumo de oxígeno por la masa libre de grasa, esas diferencias desaparecieron, lo que sugiere una función cardiovascular similar. El valor de la presión arterial diastólica aumentó durante el esfuerzo en ambos grupos, pero fue más elevado en obesos durante todo el test y alcanzó valor máximo similar en ambos grupos. La diastólica presentó elevación discreta, sin diferencia entre los grupos ${ }^{26}$.

Durante el ejercicio físico puede ocurrir un aumento superior al $200 \%$ del consumo de oxígeno por el miocardio (doble producto) debido al estiramiento de las fibras cardíacas, así como al aumento del volumen diastólico, de la presión ventricular, de la velocidad de contracción y de otros mecanismos que aún no se conocen totalmente, siendo posible correlacionar positivamente el flujo coronario y $\mathrm{MVO}_{2}$ con la frecuencia cardiaca, la presión arterial y el volumen diastólico. Los test ergométricos en cicloergómetro de brazos presentan valores más elevados del doble producto que los test en ergómetros de miembros inferiores ${ }^{11}$. Durante el test se observó un aumento del doble producto por debajo del esperado para un test máximo; sin embargo, dos factores pudieron limitar el cálculo del doble producto máximo: el hecho que la mayoría de las pacientes no consiguieron alcanzar la frecuencia cardiaca máxima prevista y la dificultad de colectar la presión arterial en el momento máximo del esfuerzo por el método de auscultación debido al esfuerzo realizado en miembros superiores.

Con el aumento de la presión arterial sistólica y de la frecuencia cardiaca durante el esfuerzo, hay disminución de duración de cada sístole, así como del periodo de diástole, generándose un menor tiempo designado para la nutrición de las fibras miocárdicas, lo que apunta a un doble producto elevado ${ }^{24}$. Luego, el doble producto es una medida indirecta del consumo de oxígeno por el miocardio y su aumento está directamente relacionado con el aumento de la presión arterial sistólica y la frecuencia cardiaca, como ya se ha descrito anteriormente en cuanto que hay una correlación del aumento de la frecuencia cardiaca con el flujo coronario y el $\mathrm{MVO}_{2}{ }^{24}$.

\section{Conclusión}

Las pacientes tuvieron buena tolerancia a la prueba de esfuerzo máximo y alcanzaron valores mayores que el $85 \%$ de la FC máxima estimada. Además, la PAS aumento con el esfuerzo y después de 5 minutos los valores no diferían significativamente de la FC de reposo. La prueba pudo incluir 
un muestreo en lugar incompatible con otro ergómetro ni se reportó dolor, así que fue posible la extracción de datos fiables para la estratificación del riesgo y la prescripción del ejercicio con el fin de respetar la individualidad biológica. Además, el equipo médico multidisciplinario del servicio de cirugía bariátrica tiene más corazón en un esfuerzo dado, además de los resultados de la prueba de 6 minutos a pie que se utiliza rutinariamente en estos pacientes. Toda prescripción clínica de ejercicio sostenido o contraindicación de la misma durante un determinado período. Los cambios cardiovasculares eran compatibles con el efecto agudo del ejercicio o condición clínica del paciente, mientras que la prueba aplicada y datos producidos fueron a la ayuda del flujo de programa de cirugía bariátrica del hospital.

Se sugiere estudios con pacientes del sexo masculino, además de la utilización de otros ergómetros.

\section{Responsabilidades éticas}

Protección de personas y animales. Los autores declaran que los procedimientos seguidos se conformaron a las normas éticas del comité de experimentación humana responsable y de acuerdo con la Asociación Médica Mundial y la Declaración de Helsinki.

Confidencialidad de los datos. Los autores declaran que en este artículo no aparecen datos de pacientes.

Derecho a la privacidad y consentimiento informado. Los autores declaran que en este artículo no aparecen datos de pacientes.

\section{Conflicto de intereses}

Ninguno.

\section{Bibliografía}

1. Daltro CS. Correlação entre o gasto energétio em repouso e composição corporal em obesos [Dissertação de Mestrado]. Salvador: Universidade Federal da Bahia; 2012.

2. Mechanick JI, Garber AJ, Handelsman Y, Garvey WT. American Association of Clinical Endocrinologists' position statement on obesity and obesity medicine. Endocr Pract. 2012;18:642-8.

3. Oliveira IV. Cirurgia bariátrica no âmbito do sistema único de saúde: tendências custos e complicações [Dissertação de Mestrado]: Universidade de Brasília; 2007.

4. Oliveira ML. Estimativa dos custos da obesidade para o sistema único de saúde do Brasil [Tese de Doutorado]: Universidade de Brasília; 2013.

5. Fornitano LD. 0 teste ergométrico em indivíduos com obesidade mórbida [Tese de Doutorado]: Faculdade de Medicina de São José do Rio Preto; 2008.

6. Fonseca-Junior SJ, Sá CGAB, Rodrigues PAF, Oliveira AJ, Fernandes-Filho J. Exercício físico e obesidade mórbida: uma revisão sistemática. Arq Bras Cir Dig. 2013;26 Supl 1:67-73.

7. Carneiro JRI. Análise de um programa de preparo pré operatório para cirurgia bariátrica com enfase na pratica regular de atividades físicas [Tese de Doutorado]: Universidade Federal do Rio de Janeiro; 2009.
8. Pouwels S, Wit M, Teijink JAW, Nienhuijs SW. Aspects of Exercise before or after Bariatric Surgery: A Systematic Review Obesity Facts. 2015;8:32-46.

9. Godoy-Matos AF, Oliveira J, Guedes EP, Carraro L, Lopes AC, Mancini MC, et al. Diretrizes brasileiras de obesidade. Associação Brasileira para o Estudo da Obesidade e da Síndrome Metabólica. 3 ed. Itapevi: AC Farmacêutica; 2009.

10. Boscatto EC, Duarte MFS, Gomes MA. Estágios de mudança de comportamento e barreiras para a atividade física em obesos mórbidos. Revista Brasileira de Cineantropometria e Desempenho Humano. 2011;13:329-34.

11. Haddad S, Ergometria de Membros Superiores. Um Método Importante na Avaliação Cardiocirculatória ao Exercício. Arquivo Brasileiro de Cardiologia. 1997;69:189-93.

12. Balady GJ, Weiner DA, Rose L, Ryan TJ. Physiologic response to arm ergometry exercise relative to age and gender. JACC Heart Fail. 1990;65:191-211.

13. Castro RR, Pedrosa S, Chabalgoity F, Sousa EB, Nobrega AC. The influence of a fast ramp rate on peak cardiopulmonary parameters during arm crank ergometry. Clin Physiol Funct Imaging. 2010;30:420-5.

14. Polito MD, Farinatti PTV. Respostas de frequência cardíaca, pressão arterial e duplo-produto ao exercício contraresistência: uma revisão da literatura. Revista Portuguesa de Ciências do Desporto. 2003;3:79-91.

15. Miranda H, Simão R, Lemos A, Dantas BHA, Baptista LA, Novaes J. Análise da freqüência cardíaca, pressão arterial e duplo-produto em diferentes posições corporais nos exercícios resistidos. Rev Bras Med Esporte. 2005;11:295-8.

16. Powers SK, Howley ET. Fisiologia do Exercício. Teoria e Aplicação ao Condicionamento e ao Desempenho. $1 \stackrel{a}{a}$ ed. Barueri-SP: Manole; 2000.

17. Neder AJ, Nery LE. Teste de Exercício Cardiopulmonar. Jornal de Pneumologia. 2002;28 Supl 3:166-206.

18. Shvartz E, Reibold RC. Aerobic fitness norms for males and females aged 6 to 75 years: a review. Aviat Space Environ Med. 1990;61:3-11.

19. Cooper CB, Storer TW. Teste ergometrico. Aplicações Práticas e

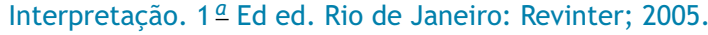

20. Meneghelo R, Araujo C, Stein S, Mastrocolla L, Albuquerque P, Serra S, et al. III Diretriz da Sociedade Brasileira de Cardiologia sobre Teste Ergométrico. Arq Bras Cardiol. 2010;95 5 (Supl 1):1-26.

21. Passaro LC. Resposta cardiovascular na prova de esforço: pressão arterial sistólica. Rev Bras Med Esporte. 1997;3:6-10.

22. Carter R, Holiday DB, Stocks J, Tiep B. Peak physiologic responses to arm and leg ergometry in male and female patients with airflow obstruction. Chest. 2003;124: 511-8.

23. Godoy M, Mantovanini JA, Santana RFT. Teste de Esforço na Mulher. Rev Soc Cardiol Estado de São Paulo. 2001;3:621-33.

24. Kawamura T. Avaliação da Capacidade física e teste ergométrico. Rev Soc Cardiol Estado de São Paulo. 2003;3:659-72.

25. Ghorayeb N, Castro I, Daher DJ, Oliveira Filho JA, Oliveira $M A B$. Diretriz em Cardiologia do Esporte e do Exercício da Sociedade Brasileira de Cardiologia e da Sociedade Brasileira de Medicina do Esporte. Arq Bras Cardiol. 2013;100 Supl 2:1-41.

26. Serés L, López-Ayerbe J, Coll R, Rodriguez O, Manresa JM, Marrugat J, et al. Cardiopulmonary Function and Exercise Capacity in Patients With Morbid Obesity. Rev Esp Cardiol. 2003;56:594-600.

27. González NF, Anchique CV, Rivas AD. Test de caminata de 6 minutos en pacientes de rehabilitación cardiaca de altitud moderada. Rev Colomb Cardiol. 2017. 